Classification

Physics Abstracts :

16.70

\title{
VERY SIMPLE METHOD FOR THE CALCULATION OF THE MEAN SQUARE DISPLACEMENT OF CRYSTAL ATOMS
}

\author{
P. MASRI $(*)$
}

Section d'Etudes des Interactions Gaz-Solides

Centre d'Etudes Nucléaires de Saclay

and L. DOBRZYNSKI $(* *)$

Physique des Solides, I. S. E. N., 3, rue François Baës, 59, Lille

(Reçu le 14 juin 1971)

\begin{abstract}
Résumé. - Nous proposons une méthode, générale simple et analytique, de calcul des déplacements carrés moyens des atomes d'un cristal. Cette méthode est testée sur un modèle de cristal cubique simple avec des interactions entre atomes premiers voisins.
\end{abstract}

Abstract. - A general analytic and very rapid method for the calculation of the mean square vibration amplitude of crystal atoms is given. This method has been tested on a simple cubic lattice with first neighbour interactions.

Low energy electron diffraction (L. E. E. D.) experiments [1] show that the intensity of the diffracted beams is a function of both the crystal temperature and the electron energy of the beams.

In accordance, and from a theoretical point of view it can be shown also [2] that the diffracted beam intensity depends upon the mean square vibration amplitude $\left\langle u^{2}\right\rangle_{T}$ of the crystal atoms where the subscript $T$ refers to the crystal temperature.

Until now the calculation of $\left\langle u^{2}\right\rangle_{T}$ has been rather tedious [3], [4], [5], [6], although it has been made either in the high or in the low temperature approximations. A general analytic and very rapid method for this calculation is given here. The accuracy of our method seems to be similar to the experimental one.

Our starting point is the following general expression for $\left\langle u^{2}\right\rangle_{T}$ as given for instance by Wallis [6] :

$<u_{k i}^{2}>_{T}=\frac{\hbar}{2 M_{k}}\left[\mathbf{D}^{-1 / 2} \operatorname{coth}\left(\hbar \mathbf{D}^{1 / 2} / 2 k_{\mathrm{B}} T\right)\right]_{k i, k i}$

where $k$ is related to the position of the atom of mass $M_{k}$ and $i$ the direction x, y, z. D is the dynamical matrix the elements of which are determined by the interactions between the atoms.

Usually the eigenvalues and the eigenvectors of $\mathbf{D}$ must be computed ; to simplify, most authors [3], [4], [5], [6] assume a few tens of atomic layers thickness for the crystal.

(*) Services de Physique Générale, Service de Physique Atomique.

$\left({ }^{* *}\right)$ Equipe de Recherches du C. N. R. S.

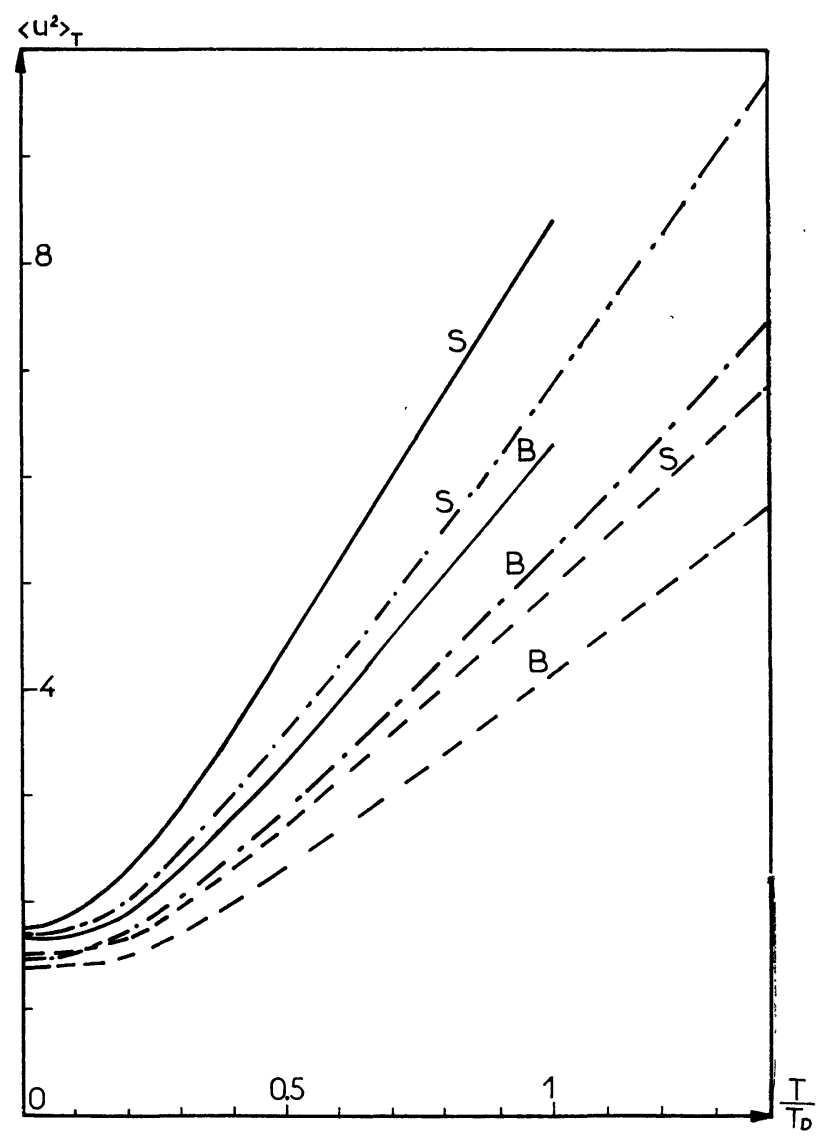

The mean-square displacement component (in units of $k_{\mathrm{B}} T_{\mathrm{D}} / 24 K$ ) along the [001] direction plotted as a function of the ratio of the crystal temperature to the crystal Debye temperature $T_{\mathrm{D}}$ for a simple cubic monoatomic crystal with a (001) free surface. $K$ is the central force constant, and $S$ and $B$ are respectively for a surface or a bulk atom - - Einstein approximation. - - - Einstein approximation with third order correction. — Rich's results. 
If, after Salter [7], we write :

$$
\mathbf{D}=\mathbf{d}+\mathbf{R}
$$

where $\mathbf{d}$ is the diagonal part of $\mathbf{D}$ and $\mathbf{R}$ the remainder part, then $\left\langle u_{k i}^{2}\right\rangle_{T}$ as given by the equation (1) can be developped in the neighbourhood of $\mathbf{d}$ and this expansion yields :

$$
\begin{aligned}
<u_{k i}^{2} & >_{T}=\frac{\hbar}{2 M_{k}}\left\{\left[\mathbf{d}^{-1 / 2} \operatorname{coth} \mathbf{a}\right]_{k i, k i}+\right. \\
& +\left(\frac{\mathbf{R}^{2}}{2}\right)_{k i, k i}\left[\frac{3}{4} \mathbf{d}^{-5 / 2} \operatorname{coth} \mathbf{a}+\frac{3}{8} \frac{\hbar}{k_{\mathrm{B}} T} \mathbf{d}^{-2} \operatorname{sh}^{-2} \mathbf{a}\right. \\
& \left.\left.+\frac{1}{8}\left(\frac{h}{k_{\mathrm{B}} T}\right)^{2} \mathbf{d}^{-3 / 2} \operatorname{ch~a~sh}^{-3} \mathbf{a}\right]_{k i, k i}+\cdots\right\}
\end{aligned}
$$

where $\mathrm{a}$ is the matrix $\hbar \mathbf{d}^{1 / 2} / 2 k_{\mathrm{B}} T$.

If $\mathbf{D} \cong \mathbf{d}$, we have the Einstein approximation. The first term of our expansion corresponds to $\left\langle u_{k i}^{2}\right\rangle_{T}$ in this approximation. The matrix elements of this expansion can be calculated by the Cyrot-Lackmann method [8]. To obtain $\left(\mathbf{R}^{2}\right)_{k i, k i}$, one needs to count the first neighbours of the $k$ atom if only first neighbour interactions are taken into account. Such a method has been previously used for the evaluation of the high temperature phonon entropy [9].
For a simple cubic lattice with first neighbour central interactions, the figure shows as a function of the temperature the values of $\left\langle u_{k i}^{2}\right\rangle_{T}$ for a $(001)$ surface atom and also for a bulk atom. Although only the [001] direction has been considered here, we must recall that, for the model we used, no anisotropy effect is expected. The computed values of Rich [3] which correspond to a rather extensive and exact calculation are reported on the same figure. When we use first to third order correction of the Einstein approximation, the accuracy for $T / T_{\mathrm{D}}=1$ is respectively 29,24 and $18 \%$ For $T / T_{\mathrm{D}}=0.2$, the corresponding values are 18,14 and $11 \%$. Thus the convergence of the series (3) is rather good, and it may be recalled here that the calculated first order accuracy is roughly of the same order of magnitude of present experimental errors.

We intend in a next paper, to apply our method to more realistic crystals models and to compare the results with experimental data.

We are indebted to Professor J. Friedel who suggested the Einstein approximation for the calculation of $\left\langle u_{k i}^{2}\right\rangle_{T}$, to Dr. Degras for his interest in this work and to Dr. J. B. Theeten for an illuminating discussion.

\section{References}

[1] MacRae (A. U.), Surf. Sci., 1964, 2, 522.

[2] Born (M.), Report Prog. Phys., 1942, 9, 294.

[3] Rich (M.), Phys. Letters, 1963, 4, 153.

[4] Maraddudin (A. A.) and Melngailis (J.), Phys. Rev., 1964, 133, A 1188.

[5] Clark (B. C.), Herman (R.) and Wallis (R. F.), Phys. Rev., 1965, 139, A 860.
[6] Wallis (R. F.), The structure and chemistry of solid surfaces, 1968, 17-1.

[7] Salter (L.), Proc. Roy. Soc., 1956, 233, 418.

[8] Cyrot-Lackmann (F.), J. Phys. Chem. Solids, 1968, 29, 1235.

[9] Dobrzynski (L.) and Friedel (J.), Surf. Sci., 1968, 12, 469. 